\title{
Buying Time: Using OMM to Potentially Reduce the Demand for Mechanical Ventilation in Patients With COVID-19
}

Michael Emerson Stenta, OMS II

\author{
Financial Disclosures: \\ None reported. \\ Support: None reported. \\ Address correspondence to \\ Michael Emerson Stenta, \\ OMS II, 215 Delhi Ave Suite \\ D, Columbus $\mathrm{OH} 43202$. \\ Email: ms861918@ohio.edu \\ Submitted \\ March 31, 2020; \\ revision received \\ April 6, 2020; \\ accepted \\ April 15, 2020. \\ The severe acute respiratory syndrome coronavirus 2 (SARS-CoV2) pandemic \\ is causing an increased need for mechanical ventilation for a significant \\ percentage of people who present to the hospital for treatment. This increase \\ in demand could surpass the supply of ventilators and lead to an increase in \\ mortality due to a lack of ventilator vacancies. There is significant evidence \\ that osteopathic manipulative medicine (OMM) can alleviate pulmonary symp- \\ toms and aid in quicker recoveries from various respiratory ailments. OMM \\ has the potential to play a significant role in helping reduce a patient's need \\ for mechanical ventilation by delaying the onset of acute respiratory distress \\ syndrome stemming from SARS-Cov2 infections. \\ J Am Osteopath Assoc. 2020;120(6):418-420. Published online May 18, 2020. \\ doi:10.7556/jaoa.2020.064 \\ Keywords: acute respiratory distress syndrome, COVID-19, osteopathic manipulative medicine, severe acute \\ respiratory syndrome
}

$\mathrm{B}$ uying patients time between symptom onset and the need for mechanical ventilation can decrease the mortality rate due to acute respiratory distress syndrome (ARDS) stemming from severe acute respiratory syndrome coronavirus 2 (SARS-CoV2) infections. Ventilator access in the United States could reach capacity and become the rate-limiting step for decreasing the coronavirus disease 2019 (COVID-19) pandemic mortality rate. ${ }^{1}$ Attempting to delay patients' need for mechanical ventilation with osteopathic manipulative medicine (OMM) may help the body to improve chest cavity expansion and aid the ability of the lymphatic vessels to drain from the lung.

One cause of mortality from SARS-CoV-2 infection stems from the accumulation of lymph in the lung's interstitium, which corresponds to overproduction of cytokines, leading to cytokine storm and pathologic changes in the lungs. ${ }^{2}$ To reduce the mortality rate caused by this virus, lymph accumulation in the lung must be reduced to aid the body's physiological mechanism for pulmonary lymphatic drainage - the expansion of the chest cavity through respiration. The respiration rate becomes more labored as ARDS progresses, activating a positive feedback loop of lymph accumulation within the lung interstitium caused by a decreased ability to breathe and expand the chest cavity. Disrupting this feedback loop will provide the body time to develop an effective adaptive immune response to SARS-CoV-2 infection, thus precluding or delaying the need for mechanical ventilation and, subsequently, increasing ventilator availability.

When used as adjunctive therapy, OMM has been shown to decrease mortality in patients with ventilator-dependent respiratory failure, ${ }^{3}$ reduce the length of pneumonia 
patients' stays in hospital, ${ }^{3}$ and reduce the duration of intravenous antibiotics in patients with pneumonia. ${ }^{4}$ OMM has also been shown to decrease shortness of breath related to activities of daily living in a patient with pulmonary fibrosis, ${ }^{5}$ and to increase lymphatic outlet flow, ${ }^{6}$ and to reduce mortality during the 1917-1918 Spanish flu pandemic. ${ }^{7}$ Patients who have received OMM have reported it as beneficial to their health and have reported a reduction in their anxiety, ${ }^{8}$ which is valuable in during the COVID-19 pandemic. As an adjunct therapy, OMM may reduce mortality by delaying patients' need for mechanical ventilation.

The following treatments are proposed as a strategy to enhance lymph drainage from the lung interstitium. Success can be maximized with implementation in the recommended order below:

1. Rib raising allows the chest cavity to move more freely and normalizes/restores the sympathetic nervous system variability via sympathetic chain ganglion activation.

2. Suboccipital release normalizes/restores parasympathetic nervous system variability via vagal stimulation to restore and maximize natural lymphatic flow.

3. Thoracic inlet technique reduces restrictions to pulmonary lymphatic outflow.

4. Thoracic pump technique. mechanically mobilizes pulmonary lymphatic fluid drainage and return to central circulation once restrictions to outflow have been reduced and normalized. This technique can be performed 2 to 3 times with a pause between cycles to allow the patient to acquire a normal breath pattern (about 30 seconds).

The following techniques also have the potential to assist patients with pulmonary-related symptoms: abdominal lymphatic pump, cervical soft tissue manipulation, dooming of the diaphragm, muscle energy of rib 1, pedal lymphatic pump, and thoracolumbar soft tissue manipulation.

Although the goal of the proposed techniques is to prevent or delay the need for mechanical ventilation, it is inevitable that some patients' symptoms will progress to a severity level for which mechanical ventilation is indicated. When a patient is intubated for mechanical ventilation, the goal of therapy is still the same: decrease lymph accumulation in the lungs by aiding the body's physiological mechanism for pulmonary lymphatic drainage - the expansion of the chest cavity through respiration. Promoting adequate lymph fluid circulation will support an adaptive immune response that can help patients return to a ventilator-free state.

The following technique is proposed by Sherman Gorbis, DO, and his associate, William J. Pintal, DO, for addressing limited rib mobility in ventilated patients in the prone position (S. Gorbis, electronic communication, May 2020):

1. Place each thumb across the T-L junction (or more superior) with fingers laterally between ribs. Apply gentle motion in various directions (1 direction at a time) toward ease. This position can be held for several seconds and then slowly and gently taken toward the directions of restriction. This maneuver can assist in the bucket handle motion of the middle to lower ribs.

2. With a soft touch, engage finger pads anteriorly under a shoulder (the patient may already be lying on 1 side, per nursing protocol) so that the sternum is accessible and place a hand across the upper- to mid-sternum and the other hand at the same level posteriorly. The anterior hand should move slowly superiorly while the posterior hand moves slowly inferiorly with patient inhalation (synchronize with the ventilator). The directions should be reversed for patient exhalation (synchronize with the ventilator). Movements can gently help enhance the pump handle motion to the upper ribs.

3. Hold the patient's elbow with a hand and the top of the same shoulder with the other hand. Slowly move the patient's upper arm superiorly and inferiorly to apply slow, gentle release to the upper ribs and surrounding tissues. 
The proposed technique is intended to assist rib mobility in both the pump handle and bucket handle motion of the predominated ribs. Assisting the body to improve chest cavity expansion can aid the ability of the lymphatic vessels to drain from the lung.

These low-risk, noninvasive, readily learned and applied techniques may be useful as an adjunctive therapy in an attempt to buy patients more time to build an effective immune response between COVID-19 symptom onset and the need for mechanical ventilation. Some of these techniques can be used when patients are mechanically ventilated in an attempt to maximize lymphatic flow, which may help them reach a ventilator-free state. It is imperative that more research be done to investigate the potential these techniques may have in reducing the demand for mechanical ventilation.

\section{Acknowledgments}

I thank the following individuals for their assistance and support: Maureen Carberry, PA-C, Shawn Kerger, DO, Scott Schefter, $\mathrm{PhD}$, Leah Sheridan, PhD, and Stevan Walkowski, DO.

\section{References}

1. Ventilator stockpiling and availability in the US. https://www. centerforhealthsecurity.org/resources/COVID-19/COVID-19-fact-sheets/ 200214-VentilatorAvailability-factsheet.pdf. Accessed May 7, 2020.

2. Cao X. COVID-19: immunopathology and its implications for therapy. Nat Rev Immunol. Published online Apr 9, 2020. doi:10.1038/ s41577-020-0308-3

3. Noll DR, Degenhardt BF, Johnson JC. Multicenter osteopathic pneumonia study in the elderly: subgroup analysis on hospital length of stay, ventilator-dependent respiratory failure rate, and in-hospital mortality rate. J Am Osteopath Assoc. 2016;116(9):574-587. doi:10.7556/jaoa.2016.117

4. Noll DR, Degenhardt BF, Morley TF, et al. Efficacy of osteopathic manipulation as an adjunctive treatment for hospitalized patients with pneumonia: a randomized controlled trial. Osteopath Med Prim Care. 2010;4:2. doi:10.1186/1750-4732-4-2

5. Goyal M, Goyal K, Narkeesh K, et al. Efficacy of osteopathic manipulative treatment approach in the patient with pulmonary fibrosis in critical care outpatient department. Indian J Crit Care Med. 2017;21 (7):469-472. doi:10.4103/0972-5229.210648

6. Knott EM, Tune JD, Stoll ST, Downey HF. Increased lymphatic flow in the thoracic duct during manipulative intervention. J Am Osteopath Assoc. 2005;105(10):447-456.

7. Smith RK. One hundred thousand cases of influenza with a death rate of one-fortieth of that officially reported under conventional medical treatment. 1919. J Am Osteopath Assoc. 2000;100(5):320-323.

8. Pomykala M, McElhinney B, Beck BL, Carreiro JE. Patient perception of osteopathic manipulative treatment in a hospitalized setting: a survey-based study. J Am Osteopath Assoc. 2008;108(11):665-668.

๑ 2020 American Osteopathic Association 